\title{
Biochar adicionado em Latossolo Vermelho beneficia o desenvolvimento de mudas de beterraba?
}

\author{
Stefany Lorrayny Lima', Ben Hur Marimon-Junior'*, Suelen Tamiozzo', \\ Fabiano André Petter², Beatriz Schwantes Marimon', Mariângela Fernandes Abreu' \\ 'Universidade do Estado de Mato Grosso, Nova Xavantina, MT, Brasil \\ ${ }^{2}$ Universidade Federal de Mato Grosso, Sinop, MT, Brasil \\ *Autor correspondente, e-mail: bhmjunior@gmail.com
}

\begin{abstract}
Resumo
O objetivo deste trabalho consistiu em verificar o efeito do biochar (carvão vegetal) adicionado em Latossolo Vermelho na produção de mudas de beterraba. O experimento foi realizado no viveiro da Universidade do Estado de Mato Grosso, município de Nova Xavantina, em 2011. 0 delineamento experimental foi inteiramente casualizado, com cinco tratamentos e quatro repetições. Foram utilizadas diferentes doses de biochar com Latossolo Vermelho avaliando-se o crescimento e a qualidade das mudas, verificando-se as diferenças entre as doses de biochar através do teste de regressão linear. A adição de biochar ao Latossolo Vermelho não promoveu acréscimo de crescimento ou qualidade das mudas de beterraba. Todos os tratamentos avaliados apresentaram desenvolvimento e qualidade das mudas abaixo do ideal. A adição de biochar de baixa qualidade química em solos de baixa fertilidade e sem adubação não é recomendada para a produção de beterraba durante o ciclo curto de produção de mudas.
\end{abstract}

Palavras-chave: Beta vulgaris, carvão vegetal, hortaliças, produção de mudas

Biochar added to a red oxisol benefits the sugar beet seedlings development?

\begin{abstract}
The objective of this study was to verify the effect of biochar (granulated charcoal) added to an oxisol on the sugar beet seedlings production. The experiment was conducted in the seedlings nursery at the Mato Grosso State University, located at Nova Xavantina municipality, MT, Brazil, during the 2011 crop year. The experiment was carried out in a completely randomized design with five treatments and four repetitions. Different doses of biochar were applied to an oxisol in order to evaluate the seedlings growth and quality, studing the differences between doses of biochar through linear regression. The biochar addition to the oxisol did not promoted growth or quality increase on sugar beet seedlings. All the evaluated treatments presented poor seedlings development and quality, below the optimal level. The addition of low-chemical quality biochar in low fertility soils and with no fertilization is not recommended for sugar beet production during the short seedling production cycle.
\end{abstract}

Keywords: Beta vulgaris, charcoal, vegetables, seedling production 


\section{Introdução}

A produção agrícola apresentou um rápido crescimento econômico e atualmente representa parte significativa da economia de muitos países, necessitando cumprir padrões de produção com qualidade, regularidade e quantidade de forma a se estabelecer no mercado.

A produção de olerícolas está entre os setores agrícola que precisam apresentar máxima eficiência para atingir a lucratividade que justifique a produção (Costa et al., 2011). Nesse sistema o substrato está entre os principais fatores que alteram a lucratividade, visto que afetam o desenvolvimento das plantas (Medeiros et al., 2008). Com isso, os produtores são altamente dependentes da disponibilidade de materiais para o preparo de substratos adequados ou de substratos comerciais que oneram a produção de olerícolas.

Visando diminuir custos de produção e a dependência de insumos comerciais como substratos e fertilizantes, o uso de biochar, ou biomassa carbonizada, na agricultura, tem sido foco de maiores estudos (Glaser et al., 2001; Lehmann, 2007; Mukome et al., 2013). O crescente interesse nesse produto se deu a partir da constatação de que a alta fertilidade das Terras Pretas de Índio na Amazônia era resultado de resíduos carbonizados de populações préColombianas (Glaser et al., 2001). Assim, a fertilidade promovida pelo descarte de resíduos carbonizados ao longo do tempo tenta ser reproduzida para a produção agrícola através da adição de biochar em solos.

Esses estudos tem verificado o potencial do biochar em melhorar as características físicas e químicas dos solos (Lehmann, 2007; Mukome et al., 2013). Sendo ainda, alternativa para pequenos produtores que podem obter 0 produto através do aproveitamento de resíduos de suas propriedades, como carvão oriundo das cozinhas (Mulcahy et al., 2013), diminuindo custos de produção e dependência dos insumos externos.

O uso do biochar na agricultura é, portanto, uma estratégia econômica e sustentável, devido ao aproveitamento de resíduos descartados no ambiente (Atkinson et al., 2010), e ao potencial de diminuição dos gastos com adubações químicas e com substratos comerciais que oneram a produção vegetal. Com isso, o objetivo deste trabalho consistiu em avaliar o efeito de diferentes doses de biochar adicionadas à Latossolo Vermelho e sua interferência no crescimento e na qualidade de mudas de beterraba (Beta vulgaris L.).

\section{Material e Métodos}

O experimento foi realizado no viveiro de mudas da Universidade do Estado de Mato Grosso (UNEMAT), município de Nova Xavantina

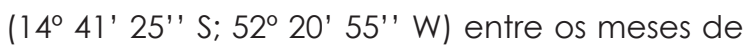
abril e maio de 2011 . Essa região apresenta clima do tipo Aw, segundo a classificação de Köppen (Silva et al., 2008), com uma estação seca, de abril a setembro, e outra chuvosa, de outubro a março.

As mudas de beterraba, cultivar Tall Top Early Wonder, foram produzidas em bandejas de poliestireno expandido de 200 células, semeando-se três sementes por célula a 1,0 cm de profundidade. As bandejas foram dispostas em bancadas sobre suporte de ferro a uma altura de 1,20 m com sombreamento de $50 \%$ e irrigação diária por microaspersão com taxa diária de aplicação ajustada conforme as condições microclimáticas observadas. Realizou-se o desbaste das plântulas aos sete dias após a semeadura (DAS) com o objetivo de manter a plântula mais vigorosa por célula.

O delineamento experimental foi inteiramente casualizado, com quatro repetições, sendo consideradas 12 plantas por repetição e utilizando células vazias entre as repetições para evitar efeitos de borda. Foram avaliadas cinco concentrações (v/v) de biochar $(0,5,10,20$ e $40 \%$ ) adicionadas ao Latossolo Vermelho, sendo as doses de biochar calculadas em relação ao volume total de substrato necessário. Foram determinadas em laboratório as características químicas dos materiais utilizados, biochar e Latossolo Vermelho (Tabela 1).

O carvão vegetal foi produzido com a carbonização parcial de madeira de eucalipto em forno de alvenaria do tipo convencional, proporcionando um ambiente pirolítico com temperatura controlada e baixas concentrações 
de oxigênio. Posteriormente o material foi moído e peneirado, apresentando característica de granulometria inferior a $0,5 \mathrm{~mm}$.

Realizou-se o tratamento prévio de ativação do biochar, com o intuito de eliminar os resíduos do processo de pirólise e desobstruir os seus poros. Para tanto, o biochar foi agitado com água em betoneira elétrica durante uma hora. A solução formada ficou em descanso por cerca de quatro dias, período após o qual o biochar foi escorrido e secado ao ar livre. A mistura do solo com o biochar foi realizada por meio de betoneira elétrica para perfeita homogeneização.

Tabela 1. Características químicas do Latossolo Vermelho e do biochar utilizados para a produção de mudas de beterraba cv. Tall Top Early Wonder.

\begin{tabular}{lccccccccc}
\hline & $\begin{array}{c}\mathrm{PH} \\
\mathrm{CaCl}_{2}\end{array}$ & $\mathrm{Ca}$ & $\mathrm{Mg}$ & $\mathrm{H}+\mathrm{Al}$ & $\mathrm{CTC}$ & $\mathrm{P}$ & $\mathrm{K}$ & $\mathrm{V}$ & $\mathrm{MO}$ \\
& 4,5 & 2,5 & 1,2 & 8,7 & 12,5 & 18,5 & 24 & 30,2 & 55,8 \\
\hline Latossolo Vermelho & 5,8 & 2,1 & 0,9 & 1,6 & 5,4 & 9,9 & 330 & 71,0 & 17,4 \\
Biochar & & &
\end{tabular}

Aos 30 dias após a semeadura (DAS) foram avaliados a altura, o diâmetro do coleto e a biomassa seca da parte aérea e do sistema radicular de 12 plantas centrais em cada repetição. Para determinação dos parâmetros de biomassa o material foi pesado em balança de precisão $(0,001 \mathrm{~g})$ após a secagem em estufa de circulação forçada de ar a $65^{\circ} \mathrm{C}$. Com os dados de massa seca da parte aérea e massa seca radicular foi determinada a massa seca total em cada tratamento

Com os resultados foram calculadas as relações entre altura e diâmetro (H/D), altura e massa seca da parte aérea (H/MSPA), massa seca da parte aérea e massa seca radicular (MSPA/MSR) e o Índice de Qualidade de Dickson, através da fórmula IQD=MST/(H/D)+(MSPA/ MSR) (Dickson et al., 1960), para efeitos de comparação da qualidade das mudas entre os tratamentos.

Para testar as diferenças entre os tratamentos quanto aos parâmetros de crescimento e qualidade das mudas, efetuou-se o estudo de regressão, com nível de significância de $5 \%$, usando o software Statistica (Statsoft, 1999).

\section{Resultados e Discussão}

Os parâmetros de crescimento das mudas de beterraba, altura, massa seca da parte aérea, massa seca radicular e massa seca total não foram influenciados estatisticamente pelos tratamentos avaliados (Tabela 2). Esses resultados demostram que a adição de biochar ao Latossolo Vermelho não beneficiou o crescimento das mudas de beterraba.

Outros estudos avaliando mudas de beterraba em diferentes tipos de substratos, como Echer et al. (2007), Silva et al. (2013) e Costa et al. (2014), demonstraram resultados superiores aos encontrados nesse trabalho para altura, com uma diferença média de $76 \%(5,5 \mathrm{~cm})$ mesmo com épocas de avaliação semelhante.

Tabela 2. Altura (H), massa seca da parte aérea (MSPA), massa seca da raiz (MSR), massa seca total (MST), Índice de Qualidade de Dickson (IQD) e relações altura/diâmetro (H/D), altura/massa seca da parte aérea (H/MSPA) e massa seca da parte aérea/massa seca radicular (MSPA/MSR) de mudas de beterraba, cv. Tall Top Early Wonder, aos 30 DAS, com a adição de doses de biochar em Latossolo Vermelho.

\begin{tabular}{ccccccccc}
\hline Biochar (\%) & $\mathrm{H}(\mathrm{cm})$ & MSPA $(\mathrm{g})$ & MSR $(\mathrm{g})$ & $\mathrm{MST}(\mathrm{g})$ & IQD & $\mathrm{H} / \mathrm{D}$ & $\mathrm{H} / \mathrm{MSPA}$ & MSPA/MSR \\
\hline 0 & 1,73 & 0,0043 & 0,0028 & 0,0072 & 0,0001 & 64,07 & 402,33 & 1,54 \\
5 & 1,74 & 0,0051 & 0,0043 & 0,0094 & 0,0002 & 58,00 & 341,18 & 1,19 \\
10 & 2,01 & 0,0042 & 0,0033 & 0,0075 & 0,0001 & 69,31 & 478,57 & 1,27 \\
20 & 1,67 & 0,0042 & 0,0035 & 0,0078 & 0,0001 & 52,19 & 397,62 & 1,20 \\
40 & 1,47 & 0,0039 & 0,0032 & 0,0071 & 0,0001 & 44,54 & 376,92 & 1,22 \\
\hline $\mathrm{P}$ & $0,21^{\text {ns }}$ & $0,25^{\text {ns }}$ & $0,85^{\text {ns }}$ & $0,52^{\text {ns }}$ & $0,58^{\text {ns }}$ & $0,08^{\text {ns }}$ & $0,83^{\text {ns }}$ & $0,37^{\text {ns }}$ \\
$\mathrm{R}^{2}$ & 0,46 & 0,40 & 0,01 & 0,15 & 0,11 & 0,69 & 0,02 & 0,27 \\
\hline ns = não significativos a 5\% de probabilidade pelos testes de regressão linear. & & & & & &
\end{tabular}


O mesmo foi verificado quanto à produção de biomassa seca da parte aérea e radicular, em que Echer et al. (2007) e Leal et al. (2011) observaram resultados com média de $94 \%(0,063 \mathrm{~g})$ acima dos que foram verificados nesse estudo. Também para a quantidade de massa seca total, os resultados de Echer et al. (2007) foram, em média, 97\% (0,27 g) superiores.

Ainda Lima et al. (2013b), avaliando - crescimento de mudas de beterraba com a adição de biochar em substratos comerciais, observaram resultados acima destes aqui verificados, com diferença média de $56 \%(2,18$ $\mathrm{cm})$ para altura, 53\% (0,004 g) para MSPA e 58\% $(0,005 \mathrm{~g})$ para MSR, em condições experimentais semelhantes a desse estudo.

Entre os parâmetros de crescimento, apenas $\bigcirc$ diâmetro apresentou diferenças estatísticas quanto à adição de biochar ao Latossolo Vermelho, onde se observou um acréscimo de $3 \%(0,001 \mathrm{~mm})$ em diâmetro a cada $10 \%$ de biochar adicionado, o que resulta em um aumento de $12 \%(0,004 \mathrm{~mm})$ com a maior dose de biochar utilizada, 40\% (Figura 1).

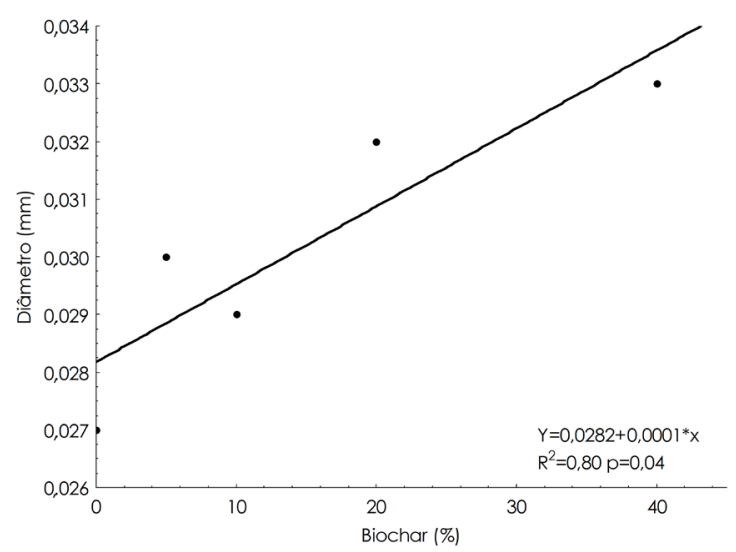

Figura 1. Diâmetro de mudas de beterraba, cv. Tall Top Early Wonder, aos 30 DAS, com a adição de doses de biochar em Latossolo Vermelho.

O uso de biochar também demostrou beneficiar o incremento em diâmetro de mudas de beterraba quando associado a um substrato comercial (Lima et al., 2013b), no entanto o resultado verificado por esses autores foi em média 60\% (0,05 mm) maior do que o verificado nesse estudo para o diâmetro das mudas.

Com isso, para o crescimento das plantas observou-se que não houve melhorias com a adição do biochar em Latossolo
Vermelho, e os substratos foram ineficientes, visto que todos os parâmetros avaliados estavam abaixo do esperado, apresentando mais de $50 \%$ de diferença em relação aos valores observados em outros estudos com diferentes arranjos de substratos.

Os resultados de qualidade das mudas de beterraba, avaliados pelo índice de Qualidade de Dickson e pelas relações H/D, H/ MSPA e MSPA/MSR, também foram semelhantes estatisticamente entre os tratamentos (Tabela 2). Os valores observados para o IQD foram em média 67\% (IQD = 0,0002) menores do que os verificados por Lima et al. (2013b) para mudas de beterraba em condições experimentais semelhantes. Também os valores da relação MSPA/MSR foram em média 53\% (1,45 g) menores do que os observados por Echer et al. (2007). Essas diferenças reforçam a hipótese de que os substratos não foram eficientes para garantir adequado crescimento e qualidade das mudas.

Para que a produção de biomassa seja eficiente precisa estar associada à nutrição adequada das plantas, o que é garantido pelo fornecimento dos nutrientes essenciais através do solo ou substrato (Fageria, 2008). Assim, o desenvolvimento abaixo do ideal das mudas de beterraba poderia estar relacionado à baixa fertilidade natural do Latossolo Vermelho utilizado para a produção das mudas (Tabela 1). Principalmente, visto que, para o crescimento adequado a beterraba apresenta alta exigência nutricional para N, P, K, Ca e Mg (Grangeiro et al., 2007; Gondim et al., 2011) e micronutrientes como Mn, Zn e Fe (Gondim et al., 2011), além de ser muito sensível à acidez do solo (Tivelli et al., 2011). Ainda a maior produção de biomassa nessa espécie está diretamente relacionada com o suprimento adequado principalmente de N (Trani et al., 2005; Tivelli et al., 2011) e P (Avalhaes et al., 2009).

Como as características químicas do Latossolo não foram alteradas a fim de verificar possíveis respostas da adição do biochar, a capacidade de nutrição das plantas foi limitada pela baixa fertilidade da mistura utilizada como substrato. No entanto, esperava-se que a adição de biochar promovesse acréscimo no desenvolvimento vegetal, visto que em 
geral, a literatura aponta esse produto como um condicionante que promove a melhoria das condições físico-químicas do solo e consequentemente do rendimento da cultura (Lehmann, 2007; Gaskin et al., 2010).

Ainda, alguns estudos verificaram que o biochar age como fertilizante, adicionando quantidade considerável de alguns nutrientes ao solo ou substrato, como o $\mathrm{Ca}, \mathrm{Mg}, \mathrm{P}$ e $\mathrm{K}$ (Altland \& Locke, 2012; Carter et al., 2013). Isso sustentaria a hipótese de que a adição do produto em um substrato base resultaria em benefício no crescimento vegetal. Também nesse estudo verificou-se que o biochar adicionado apresentava altas concentrações de $K$ (Tabela 1), mas não houve interferência no desenvolvimento das plantas, provavelmente devido ao desbalanço nutricional promovido pelo suprimento inadequado dos outros elementos essenciais.

Além disso, o potencial do biochar em melhorar as condições nutricionais dos solos varia conforme as características do produto, afetadas pelos tipos de matéria prima e de formas de produção, e do tipo de solo ou substrato utilizado (Mukherjee \& Zimmerman, 2013; Mukome et al., 2013). Isso acarreta em características distintas e resultados muito variados em estudos com o produto.

Devido ao potencial de uso do biochar e da diversidade de resultados encontrados com produtos obtidos de diferentes matérias primas e formas de produção faz-se necessário novos estudos que identifiquem as características do solo e do biochar para utilizá-lo conforme cada condição e objetivo, proporcionando resultados que possam direcionar o produto para uso em técnicas de produção agrícola.

Ainda, segundo Petter et al. (2012), os resultados também podem apresentar diferenças devido à longevidade do material no solo, o que pode influenciar o processo de produção de mudas de olericola, que tem um ciclo bastante rápido. Assim, o biochar pode apresentar melhor desempenho na produção de olerícolas após o transplante das mudas, devido ao maior tempo de reação do produto, beneficiando o desenvolvimento das culturas. Estudos que avaliem o uso desse produto durante toda a fase de produção de diferentes espécies de olerícolas podem direcionar o uso eficiente do biochar nesse setor produtivo.

Outros estudos também discutem que alguns tipos de biochar não atuam como fonte de nutrientes, devido à baixa quantidade de elementos em sua composição (Winsley, 2007; Lima et al., 2013a). Assim sua ação se dá apenas na melhoria das características físicas dos solos e substratos, o que auxilia na retenção dos elementos que já estão disponíveis no material principal. Com isso, o substrato base pode influenciar na quantidade de nutrientes disponíveis e, consequentemente, afetar a eficiência do produto e o desenvolvimento vegetal.

O potencial do biochar em atuar no benefício das características físicas do solo e substrato é sustentado por alguns estudos que demonstram maior desenvolvimento e produtividade vegetal quando $\circ$ produto é adicionado em conjunto com um solo ou substrato base quimicamente adequados (Altland \& Locke, 2012; Marimon-Junior et al., 2012; Lima et al., 2013b). Isso sugere a necessidade de identificar as características do biochar antes de justificar seu uso como fonte de nutrientes ou com a necessidade de adicionálo a um substrato quimicamente adequado. Isso poderá direcionar o uso prático do mesmo em sistemas de produção e incentivar sua produção sustentável para uso agrícola.

\section{Conclusões}

A adição de biochar ao Latossolo Vermelho sem fertilização não promove incremento no crescimento e na qualidade das mudas de beterraba.

Novos testes com biochar e substratos quimicamente adequados para a nutrição vegetal são sugeridos a fim de verificar a eficiência do produto em técnicas de produção de mudas.

Considerar o uso de biochar com maior quantidade de nutrientes e a avaliação em maior período de tempo pode apresentar respostas favoráveis à produção agrícola em novos estudos. 


\section{Referências}

Altland, J.E., Locke, J.C. 2012. Biochar affects macronutrient leaching from a soilless substrate. Hortscience 47: 1136-1140.

Atkinson, C.J., Fitzgerald, J.D, Hipps, N.A. 2010. Potential mechanisms for achieving agricultural benefits from biochar application to temperate soils: a review. Plant and Soil 337: 1-18.

Avalhaes, C.C., Prado, R.M., Gondim, A.R.O., Alves, A.U., Correia, M.A.R. 2009. Rendimento e crescimento da beterraba em função da adubação com fósforo. Scientia Agraria 10: 075080 .

Carter, S., Shackley, S., Sohi, S., Suy, T.B., Haefele, S. 2013. The impact of biochar application on soil properties and plant growth of pot grown lettuce (Lactuca sativa) and cabbage (Brassica chinensis). Agronomy 3: 404-418.

Costa, E., Durante, L.G.Y., Nagel, P.L., Ferreira, C.R., Santos, A. 2011. Qualidade de mudas de berinjela submetida a diferentes métodos de produção. Revista Ciência Agronômica 42: $1017-$ 1025.

Costa, L.A.M., Pereira D.C., Costa, M.S.S.M. 2014. Substratos alternativos para produção de repolho e beterraba em consórcio e monocultivo. Revista Brasileira de Engenharia Agrícola e Ambiental 18: 150-156.

Dickson, A., Leaf, A.L., Hosner, J.F. 1960. Quality appraisal of white spruce and white pine seedling stock in nurseries. Forest Chronicle 36: 10-13.

Echer, M.M., Guimarães, V.F., Aranda, A.N., Bortolazzo, E.D., Braga, J.S. 2007. Avaliação de mudas de beterraba em função do substrato e do tipo de bandeja. Semina: Ciências Agrárias 28: $45-50$.

Fageria, N.K. 2008. The use of nutrients in crop plants. CRC Press, New York, EUA. 428 p.

Gaskin, J.W., Speir, R.A., Harris, K., Das, K.C., Lee, R.D., Morris, L.A., Fisher, D.S. 2010. Effect of peanut hull and pine chip biochar on soil nutrients, corn nutrient status, and yield. Agronomy Journal 102: 623-633.

Glaser, B., Haumaier, L., Guggenberger, G., Zech, W. 2001. The 'Terra Preta' phenomenon: a model for sustainable agriculture in the humid tropics. Naturwissenschaften 88: 37-41.

Gondim, A.R.O., Correia, M.A.R., Alves, A.U., Prado, R.M., Cecílio Filho, A.B. 2011 . Crescimento e marcha de acúmulo de nutrientes em plantas de beterraba cultivadas em sistema hidropônico. Bioscience Journal 27: 526-535.

Grangeiro, L.C., Negreiros, M.Z., Souza, B.S.,
Azevêdo, P.E., Oliveira, S.L., Medeiros, M.A. 2007. Acúmulo e exportação de nutrientes em beterraba. Ciência e Agrotecnologia 31: 267273.

Leal, P.A.M., Costa, E., Schiavo, J.A., Pegorare, A.B. 2011 . Seedling formation and field production of beetroot and lettuce in Aquidauana, Mato Grosso do Sul, Brazil. Horticultura Brasileira 29: 465471.

Lehmann, J. 2007. Bio-energy in the black. Frontiers in Ecology and the Environment 5: 381387.

Lima, S.L., Marimon-Junior, B.H., Petter, F.A., Tamiozzo, S., Buck, G.B., Marimon, B.S. 2013. Biochar as substitute for organic matter in the composition of substrates for seedlings. Acta Scientiarum. Agronomy 35: 333-341.a.

Lima, S.L., Tamiozzo, S., Petter, F.A., Marimon, B.S., Marimon-Junior, B.M. 2013. Desenvolvimento de mudas de beterraba em substratos comerciais tratados com biochar. Agrotrópica 25: 181-186.b.

Marimon-Junior, B.H., Petter, F.A., Andrade, F., Madari, B.E., Marimon, B.S., Schossler, T.R., Goncalves, L.G.V., Belém, R.S. 2012. Produção de mudas de jiló em substrato condicionado com Biochar. Comunicata Scientiae 3: 108-114.

Medeiros, D.C., Freitas, K.C.S., Veras, F.S., Anjos, R.S.B., Borges, R.D., Cavalcante Neto, J.G., Nunes, G.H.S., Ferreira, H.A. 2008. Qualidade de mudas de alface em função de substratos com e sem biofertilizante. Horticultura Brasileira 26: 186-189.

Mukherjee, A., Zimmerman, A.R. 2013. Organic carbon and nutrient release from a range of laboratory-produced biochars and biochar-soil mixtures. Geoderma 194: 122-130.

Mukome, F.N.D., Zhang, X., Silva, L.C.R., Six, J., Parikh, S.J. 2013. Use of chemical and physical characteristics to investigate trends in biochar feedstocks. Journal of Agricultural and Food Chemistry 61: 2196-2204.

Mulcahy, D.N., Mulcahy, D.L., Dietz, D. 2013. Biochar soil amendment increases tomato seedling resistance to drought in sandy soils. Journal of Arid Environments 88: 222-225.

Petter, F.A., Madari, B.E., Soler, M.A.S., Carneiro, M.A.C., Carvalho, M.T.M., Marimon-Junior, B.H., Pacheco, L.P. 2012. Soil fertility and agronomic response of rice to biochar application in the Brazilian savannah. Pesquisa Agropecuária Brasileira 47: 699-706.

Silva, F.A.M., Assad, E.D., Evangelista, B.A. 2008. Caracterização climática do Bioma Cerrado. In: Sano, S.M., Almeida, S.P., Ribeiro, J.F. (ed.) Cerrado: ecologia e flora. Embrapa, Planaltina, 
Brasil. p. 69-88.

Silva, F.C. da. Manual de análises químicas de solos, plantas e fertilizantes. Brasília: Embrapa Comunicação para Transferência de Tecnologia, 1999. 370p

Silva, F.F., Gondim, A.R.O., Viera, A. R., Francilino, A.H., Silva, Y.A., Silva, J.L.B. 2013. Uso de substratos e suas combinações na produção de mudas de alface e beterraba no Iguatu-CE. Agropecuária Científica no Semiárido 9: 10-16.

Statsoft Inc. 1999. Statistica for WindowsComputer program manual. StatSoft, Tulsa, USA.

Tivelli, S.W., Factor, T.L., Teramoto, J.R.S., Fabri, E.G., Moraes, A.R.A., Trani, P.E., May, A. 2011. Beterraba: do plantio à comercialização. IAC, Campinas, Brasil. $51 \mathrm{p}$.

Trani, P.E., Cantarella, H., Tivelli, S.W. 2005. Produtividade de beterraba em função de doses de sulfato de amônio em cobertura. Horticultura Brasileira 23: 726-730.

Winsley, P. 2007. Biochar and bioenergy production for climate change mitigation. Science Review 64: 5-10. 